\title{
Does the entropy of the Universe tend to a maximum?
}

\author{
Diego Pavón* and Ninfa Radicella ${ }^{\dagger 1}$ \\ ${ }^{1}$ Departamento de Física, Universidad Autónoma \\ de Barcelona, 08193 Bellaterra (Barcelona), Spain.
}

\begin{abstract}
Ordinary, macroscopic systems, naturally tend to a state of maximum entropy compatible with their constraints. However, this might not hold for gravity-dominated systems since their entropy may increase without bound unless this is precluded by the formation of a black hole. In this short note we suggest, based on the Hubble expansion history, that our Universe likely behaves as an ordinary system, i.e., that its entropy seems to tend to some maximum value.
\end{abstract}

\footnotetext{
* E-mail: diego.pavon@uab.es

† E-mail: ninfa.radicella@uab.cat
} 
As is well known, systems dominated by electromagnetic forces spontaneously tend to some equilibrium state compatible with the constraints the system is subjected to. This constitutes the hard core of the empirical basis of the second law of thermodynamics. According to the latter isolated, macroscopic systems, evolve to the maximum entropy state consistent with their constraints [1]. This implies two separate things: first, the entropy, $S$, of isolated systems cannot decrease, i.e., $S^{\prime} \geq 0$, where the prime means derivative with respect to the relevant, appropriate variable. Secondly, it must be a convex function of the said variable, $S^{\prime \prime}<0$, at least at the last stage of the evolution.

However, this is not necessarily true when gravity plays a significant role. The entropy of systems dominated by gravity must still increase (as formulated by the generalized second law of thermodynamics [2 4] $]$ ), but -at least in Newtonian gravity- it may grow unbounded. That is to say, while the relationship $S^{\prime}>0$ remains in place it may well happen that $S^{\prime \prime}>0$ when the variable approaches its final value; in such a case, no maximum entropy state would be achievable. This is illustrated by the gravothermal catastrophe; namely, the final stage of $N$ gravitating point masses enclosed in a perfectly reflecting, rigid, sphere whose radius exceeds some critical value $[5,6]$.

Nevertheless, when Newtonian gravity is replaced by general relativity a black hole is expected to form at the center of the sphere whereby the said catastrophe is prevented and the entropy does not diverge. Though the black hole will tend to evaporate, which will again increase the total entropy, it will likely arrive to an equilibrium state with its own radiation -see e.g. [7]- whence the end stage of the whole process will be characterized by a state of maximum, finite, entropy.

At any rate, as far as we know, the possibility of realistic processes in which no equilibrium state is achievable, because $S^{\prime \prime}>0$ in the long run, cannot be ruled out right away. Then, the question arises whether the entropy of the Universe mimics the first set of systems (ordinary systems) or the second -exotic- set. The aim of this short note is to shed light on this issue. As we shall see, our analysis suggests that it mimics the entropy of systems falling in the first set, i.e., in this respect, the Universe behaves as an ordinary system. To make matters simpler we will work in the framework of general relativity and assume the Universe sufficiently well described at large scales by the spatially-flat Friedmann-Robertson-Walker (FRW) metric of scale factor $a$. 
We shall base our study, on the one hand, on the close connection between entropy and area of cosmic horizons and, on the other hand, on observational results about the history of the Hubble factor, $H=\dot{a} / a$, of the FRW metric. While these results are still rather preliminary, it seems beyond doubt that $H$ decreases with expansion, i.e., $H^{\prime}(a)<0$, where the prime means derivative with respect to $a$, and that $H^{\prime \prime}(a)>0$. This is fully consistent with recent studies on the impact of hypothetical transient periods of acceleration-deceleration on the matter growth [8] and on the radiation power spectrum [9] from the decoupling era, $a \simeq 10^{-5}$, to $a=0.5$, and with the study of Serra et al. [14] that shows that equation of state parameter of dark energy has not been noticeably varied between $a=0.5$ and 1 , where the normalization $a_{0}=1$ is understood. These studies strongly suggest the absence of the said hypothetical periods. A further and crucial observation is that the cosmic expansion is accelerating at present [10 13], thereby the current value of the deceleration parameter results negative, $q_{0}=-\left[1+\left(a H^{\prime} / H\right)\right]_{0}<0$.

Nowadays it is widely accepted that, sooner or later, the entropy of the expanding Universe is to be dominated by the entropy of the horizon, which is proportional to the area of the latter, i.e., $S \propto \mathcal{A}$. This relation is valid, at least, in Einstein gravity [15]. We will deal here with apparent horizons that are endowed with thermodynamical properties, formally identical to those of event horizons [17, 18]. The apparent horizon is defined as the marginally trapped surface with vanishing expansion of radius $\tilde{r}=a(t) r[16]$. In the simplest case of a spatially flat FRW universe $\tilde{r}_{A}=H^{-1}$ and

$$
\mathcal{A} \propto H^{-2}
$$

Assuming that the Universe expands for ever (i.e., $H>0$ at all times) and behaves from the thermodynamical standpoint as any other macroscopic system, it is natural to expect that it approaches equilibrium, characterized by a state of maximum entropy compatible with the constraints. This translates into the inequalities, $\mathcal{A}^{\prime}>0$ at all times and $\mathcal{A}^{\prime \prime}<0$ at least as $a \rightarrow \infty$.

From (1) we have

$$
\mathcal{A}^{\prime} \propto-\frac{2 H^{\prime}}{H^{3}}, \quad \text { and } \quad \mathcal{A}^{\prime \prime} \propto \frac{2}{H^{2}}\left[3\left(\frac{H^{\prime}}{H}\right)^{2}-\frac{H^{\prime \prime}}{H}\right] .
$$

Measurements of the Hubble factor at different redshifts [19, 20] plus numerical simulations 21, 22] fairly suggest that $H^{\prime}(a)<0$ and $H^{\prime \prime}(a)>0$, at least in the interval $0.4 \leq a \leq 1$-see Figs. 1(c) and 3(a) in [22], and Fig. 1 below. The latter shows the projected evolution of the 
Hubble function in terms of the scale factor in the said interval; the set of points was adapted from Fig. 3(a) of [22], which results from numerical simulations -assuming a precision of 1\%of $\mathrm{H}(\mathrm{z})$ measurements from luminous red galaxies [21], plus the recently measured value of the Hubble constant, $H_{0}=74.2 \pm 3 \mathrm{Km} / \mathrm{s} / \mathrm{Mpc}$-see Riess et al. [23].

The overall behavior these figures show is shared by the spatially flat $\Lambda \mathrm{CDM}$ model which seems to pass fairly well most, if not all, observational tests. This implies that whatever the "right" cosmological model turns out to be, it will not substantially differ, observationally, from the $\Lambda$ CDM. Since there is no apparent reason for this trend to change in the future (it would if the Universe expansion were dominated by phantom dark energy) we shall assume that the inequalities of above will stay in place also for $a>1$. In consequence $\mathcal{A}^{\prime}$ will result positive-definite, however $\mathcal{A}^{\prime \prime}$ may bear any sign. By imposing that $\mathcal{A}^{\prime \prime}$ should be negative, the constraint

$$
3\left(\frac{H^{\prime}}{H}\right)^{2}<\frac{H^{\prime \prime}}{H}
$$

readily follows.

From the set of conditions $H^{\prime}<0, H^{\prime \prime}>0$, and $q<0$-the latter holding only from some "recent time" on-, it can be demonstrated that for sufficiently large scale factor onwards the inequality (3) is to be satisfied and, accordingly, $\mathcal{A}^{\prime \prime}<0$. Effectively, bear in mind that $q=-\left[1+\left(a H^{\prime} / H\right)\right]$; then $H^{\prime} / H=-(1+q) / a$. Since $H^{\prime}$ and $q$ are negative the numerator of last expression stays bounded (it lies in the range $0 \leq 1+q \leq 1$ ), whence the left hand side of (3) vanishes in the long run.

Inspection of panels (c) of Fig. 1 and (d) of Fig. 3 in [22], as well as Fig. 1 below, suggests that the data points can be roughly approximated by the simple expressions

$$
H=H_{*} \exp (\lambda / a) \quad \text { and } \quad H=H_{*}\left(1+\lambda a^{-n}\right),
$$

where $H_{*}=H(a \rightarrow \infty)>0, \lambda>0$, and $n>1$. Both functions describe ever expanding universes with $H^{\prime}<0$ and $H^{\prime \prime}>0$. By inserting the first one in (3) one obtains that $\mathcal{A}^{\prime \prime}<0$ from the instant the Universe starts accelerating onwards, namely, for $a \geq \lambda$. By fitting (4,1) to the set of points displayed in Fig. 1 (dashed line) we find that $H_{*}=42.6 \pm 0.4 \mathrm{~km} / \mathrm{s} / \mathrm{Mpc}$ and $\lambda=0.550 \pm 0.005$, both at 95\% confidence level (CL).

For the cosmic expansion described by (4,2) the transition from deceleration to acceleration occurs 
when $a_{t r}=[\lambda(n-1)]^{1 / n}$, and $\mathcal{A}^{\prime \prime}<0$ for $a>\left[\lambda\left(\frac{3 n}{n+1}-1\right)\right]^{1 / n}$. The best fit values, at $95 \%$ $\mathrm{CL}$, of the parameters to the set of points in Fig. 1 are $H_{*}=54.78 \pm 0.06 \mathrm{~km} / \mathrm{s} / \mathrm{Mpc}, \lambda=$ $0.3535 \pm 0.0013$ and $n=1.928 \pm 0.002$-see the dot-dashed line in the said figure. For completeness and comparison we have also drawn the curve corresponding to spatially flat $\Lambda$ CDM model, $H(a)=$ $H_{0} \sqrt{\Omega_{m 0} a^{-3}+\left(1-\Omega_{m 0}\right)}$ with $H_{0}=73.3 \mathrm{Km} / \mathrm{s} / \mathrm{Mpc}$ and $\Omega_{m 0}=0.29$ which follow from the fit to the set of points (solid line).

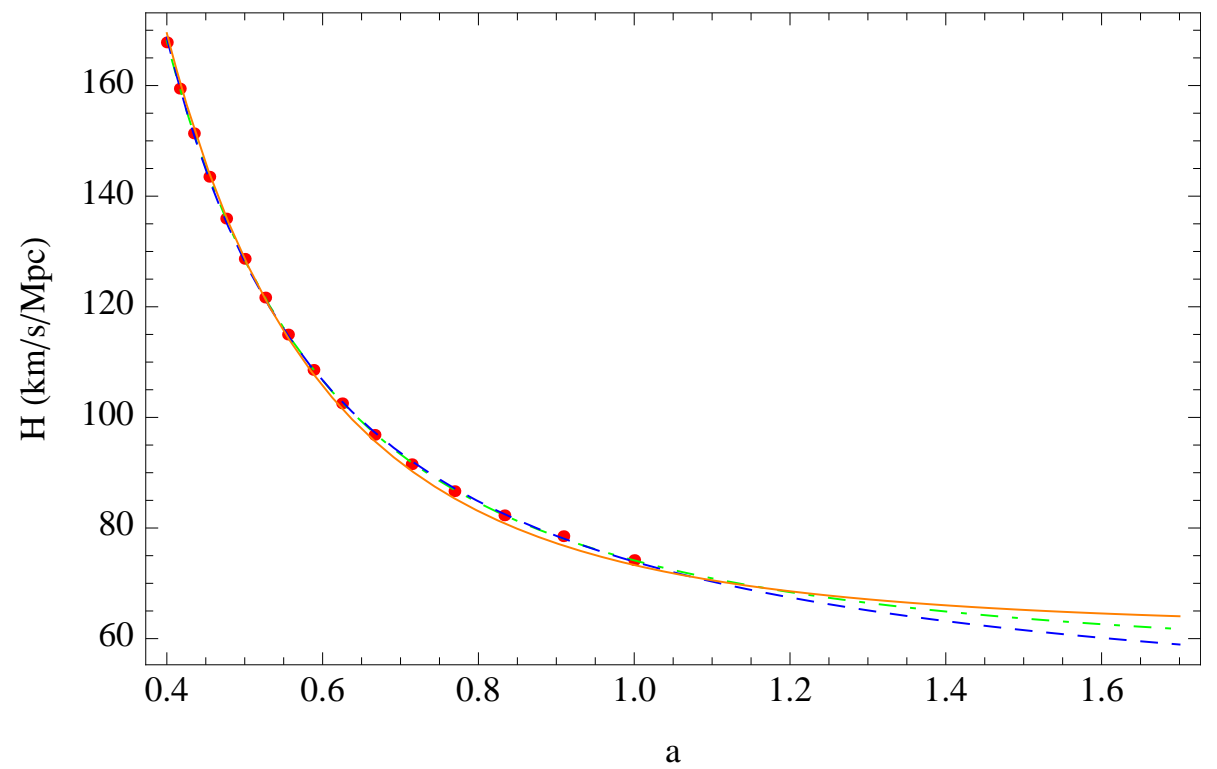

Figure 1: The string of points show the Hubble history in the interval $0.4 \leq a \leq 1$. The one at $a=1$ indicates the Hubble constant value, $H_{0}$, as measured by Riess et al. 23]. The other fourteen points correspond to simulated values of the Hubble function assuming an accuracy of $1 \%$ in the $H(a)$ observations according to Carvalho and Alcaniz (Fig. 3 (a) in Ref. [22]). The dashed, dot-dashed, and solid lines are the best fit curves of the models represented by Eq. (4,1), Eq. (4.2), and the spatially flat $\Lambda$ CDM model, respectively.

For completeness, Fig. 2 presents the evolution of deceleration parameter of the models considered. Though not shown, in all the cases $q(a \rightarrow \infty)=-1$.

At this point it is sobering to note that not all Hubble functions that fulfill the observational restrictions $H^{\prime}<0$ and $H^{\prime \prime}>0$ comply with the inequality (3). This is, for instance, the case of the expansion laws $H=H_{*}\left[\exp \left(\lambda a^{-1}\right)-1\right]$ and $H=H_{*} \exp (-\lambda a)$ (with $\left.\lambda>0\right)$. Clearly, the entropy of a universe that obeyed any of these two laws would increase without bound in the 
long run, similarly to the entropy of Antonov's sphere in Newtonian gravity. Note, however, that the said functions do not correspond to realistic universes. In the first case the universe never accelerates; in the second one the universe accelerates at early times (when $a<1 / \lambda$ ) to decelerate for ever afterwards. Thus, both of them are grossly incompatible with observations. This suggests that Hubble functions that satisfy the constraints $H^{\prime}<0$ and $H^{\prime \prime}>0$ but violate Eq. (3) (thereby leading an unbound entropy as $a \rightarrow \infty$ ) should be dismissed.

Finally, as it can be checked, Eq. (3) would be satisfied by quintessence-like dark energy models but not by phantom dominated models [24, 25]. Moreover, dynamical models that tend to de Sitter expansion at late times approach equilibrium since the entropy function shows an horizontal asymptote, a maximum entropy equilibrium state, towards which the system tends from below. We have not considered the entropy of matter and/or fields driving the expansion of the Universe. The reasons for this are as follows. ( $i$ ) As said above, except for the case of phantom fields, these are subdominant in the long run whence, in any case, the entropy of the horizon will eventually prevail. Things are, nonetheless, different in the case of phantom fields. They present the feature $S^{\prime \prime}>0$ and could invalidate our argument if they drove the accelerated expansion. However, as is well known, they suffer from inherent quantum instabilities [26, 27] whereby they can hardly

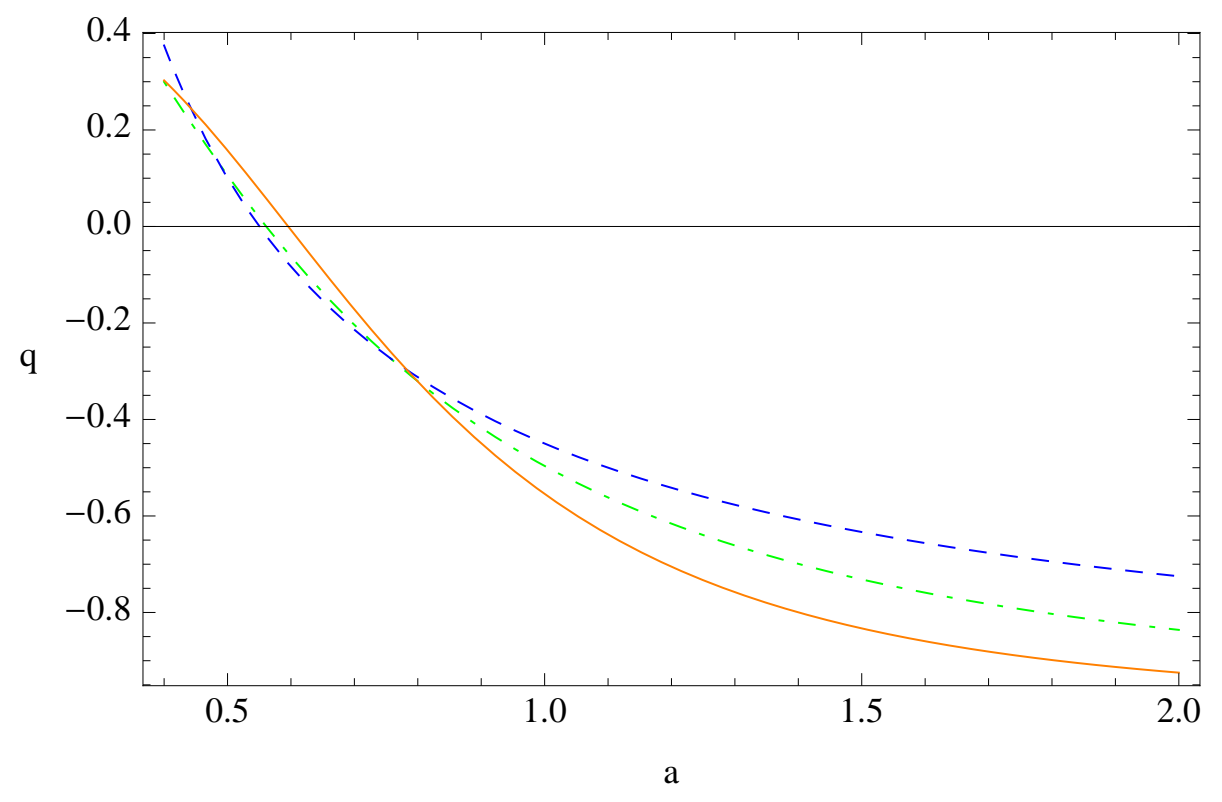

Figure 2: The deceleration parameter, $q=-\left(1+a H^{\prime} / H\right)$, as a function of the scale factor. The dashed, dot-dashed, and solid lines correspond to the models of Eq. (41), Eq. (4)2), and the spatially flat $\Lambda$ CDM model, respectively. 
be considered serious candidates for dark energy; this is why we ignored them. (ii) The entropy generated at small scales by matter and or radiation through a variety of dissipative processes obeys the second law of thermodynamics; taking it into account would only strengthen our argument.

Altogether, we may tentatively conclude that the entropy of the Universe -like that of any ordinary system-, rather than to increase indefinitely (as is the case of Antonov's sphere), appears to tend to some maximum value, possibly of the order of $H^{-2}$ when $a \rightarrow \infty$. To reach a firmer conclusion on this issue more abundant and accurate measurements regarding the Hubble history of the Universe are needed.

\section{Acknowledgments}

We are grateful to Fernando Atrio-Barandela and José Gaite for constructive remarks. NR is funded by the Spanish Ministry of Education through the "Subprograma Estancias de Jóvenes Doctores Extranjeros, Modalidad B", Ref: SB2009-0056. This work was partly supported by the Spanish Ministry of Science and Innovation under Grant FIS2009-13370-C02-01, and the "Direcció de Recerca de la Generalitat" under Grant 2009SGR-00164.

[1] H.B. Callen, Thermodynamics (J. Wiley, N.Y., 1960).

[2] J. Bekenstein, Phys. Rev. D $\underline{9}, 3292$ (1974).

[3] J. Bekenstein, Phys. Rev. D $\underline{12}, 3077$ (1975).

[4] G.L. Sewell, Phys. Lett. A $\underline{122}, 309$ (1987).

[5] V.A. Antonov, Vest. leningr. gos. Univ. ㄱ, 135 (1962).

[6] D. Lynden-Bell and R. Wood, Mon. Not. R. Astron. Soc. $\underline{138}, 495$ (1968).

[7] K.S. Thorne, W.H. Zurek, and R.H. Price, "The thermal atmosphere of a black hole", in Black Holes: The Membrana Paradigm, edited by K.S. Thorne, R.H. Price and D.A. Macdonald (Yale University Press, New Haven, 1986).

[8] E.V. Linder, Phys. Rev. D $\underline{82}, 063514$ (2010).

[9] E.V. Linder and T.L. Smith, JCAP 04(2011)001.

[10] A.G. Riess et al., Astron. J. 116, 1009 (1998).

[11] S. Perlmutter et al., Astrophys. J. $\underline{517}, 565$ (1999).

[12] E. Komatsu et al., Astrophys. J. Suppl. Ser. 180, 330 (2009).

[13] R. Amanullah et al., Astrophys. J. $\underline{716}, 712$ (2010). 
[14] P. Serra et al., Phys. Rev. D $\underline{80}, 121302$ (2009).

[15] G. Gibbons and S.W. Hawking, Phys. Rev. D $\underline{15}, 2738$ (1977).

[16] D. Bak and S.-J. Rey, Class. Quantum Grav. $\underline{17}$, L83 (2000).

[17] R.-G. Cai, Progr. Theoret. Phys. Suppl. 172, 100 (2008).

[18] R.-G. Cai, L.-M. Cao, and Y.-P. Hu, Class. Quantum Grav. 26, 155018 (2009).

[19] J. Simon et al., Phys. Rev. D. $\underline{71}, 123001$ (2005).

[20] D. Stern et al., JCAP 02(2010)008.

[21] S.M. Crawford et al., Mon. Not. R. Astron. Soc. $\underline{406}, 2569$ (2010).

[22] J.C. Carvalho and J.S. Alcaniz, arXiV: 11025319 [astro-ph.CO].

[23] A.G. Riess et al., Astrophys. J. $\underline{699}, 539$ (2009).

[24] N. Radicella and D. Pavón, Gen. Relativ. Grav. $\underline{44}, 685$ (2012).

[25] N. Radicella and D. Pavón, Phys. Lett. B $\underline{704}, 206$ (2011).

[26] S.M. Carroll, M. Hoffman, and M. Trodden, Phys. Rev. D $\underline{68}, 023509$ (2003).

[27] J.M. Cline, S. Jeon, and G.D. Moore, Phys. Rev. D 므, 043543 (2004). 